\title{
Prioritized Brain Circulation During Ergometer Cycling with Apnea and Face Immersion in Ice-Cold Water: A Case Report
}

\author{
Lars J Bjertnaes ${ }^{1,2}$ \\ Anton Hauge ${ }^{3}$ \\ Marianne Thoresen (1D ${ }^{3,4}$ \\ Lars Walløe (D) ${ }^{3}$
}

'Anesthesia and Critical Care Research Group, Department of Clinical Medicine, Faculty of Health Sciences, UiT The Arctic University of Norway, Tromsø, N-9037, Norway; ${ }^{2}$ Department of Intensive Care Medicine, University Hospital of North Norway, Tromsø, N9017, Norway; ${ }^{3}$ Division of Physiology, Institute of Basic Medical Sciences, University of Oslo, Oslo, 0317, Norway; ${ }^{4}$ Translational Health Sciences, University of Bristol, Bristol, UK
Correspondence: Lars J Bjertnaes Anesthesia and Critical Care Research Group, Department of Clinical Medicine, Faculty of Health Sciences, UiT The Arctic University of Norway, Tromsø, N-9037, Norway

Tel +477762700 I

Fax +4777626042

Email lars.bjertnaes@uit.no
Background: Successful cardiopulmonary resuscitation after drowning or avalanche is often attributed to hypothermia-induced decrease in metabolism, which adapts the oxygen demand to the amount supplied under cardiac compression. Four decades ago, we speculated if oxygen-sparing mechanisms like those found in marine mammals, may improve cerebral oxygenation during acute airway blockade in humans. We investigated hemodynamic changes during steady state ergometer cycling with intermittent periods of apnea and face immersion (AFI) in ice-cold water. During AFI, heart rate (HR) dropped by $58 \%$ whereas average blood velocity (ABV) determined by means of a Doppler ultrasound velocity meter (UNIDOP University of Oslo, Oslo, Norway) fell by $85 \%$ in the radial artery and rose by $67 \%$ in the vertebral artery. Similar changes occured in radial artery ABV, albeit more slowly, when the test subject only held his breath while cycling. When he breathed via a snorkel during face immersion, HR remained unchanged while radial artery ABV fell transiently and subsequently returned to its pre-immersion level. These findings later were confirmed by other investigators. Moreover, a recent study revealed that the seal even has a system for selective brain cooling during the dive.

Conclusion: Our research has confirmed prioritized cerebral circulation during AFI in cold water. We hypothesize that these changes may improve brain oxygenation due both to greater blood flow and possibly also to faster brain cooling, as demonstrated in diving seals.

Keywords: apnea, breath-hold, cardiopulmonary resuscitation, diving response, hypothermia

\section{Introduction}

Throughout more than half a century, physicians have reported successful cardiopulmonary resuscitations after lengthy periods of asphyxia and cardiac arrest following drowning and avalanche. ${ }^{1,2}$ Most authors ascribe their success to hypothermia-induced decrease in metabolism, which adjusts oxygen consumption to the supply being delivered during cardiac compression. According to Fodden et al, trained personnel using standard cardiopulmonary resuscitation techniques were able to obtain cardiac output (CO) values consistent with those observed during rest in human. ${ }^{3}$

In 1980, we speculated whether oxygen-saving mechanisms of marine mammals, that had been demonstrated in noninvasive experiments on humans, possibly could contribute to prevent anoxic brain injury under conditions of inhibited breathing in man. ${ }^{4,5}$ In a heart catheterization study, while exercising at constant 
workload on an ergometer cycle, two male subjects underwent periods of apnea and face immersion (AFI) as long as they could endure (30-45 seconds) in a basin filled with ice-cold water.

During AFI, heart rate (HR) fell from maximums obtained during steady state cycling of 160 and 150 beats/min, to nadirs of 40 and 50 beats/min, respectively. Concomitantly, CO fell by approximately 50\% from 24 and $18 \mathrm{~L} / \mathrm{min}$, respectively. Despite a rise in systemic vascular resistance by nearly $200 \%$, myocardial oxygen demand, determined as the HR pressure product, decreased by $42 \%{ }^{6}{ }^{6}$ These findings inspired us to perform a hypothesis-forming experiment during ergometer cycling with periods of AFI at which we compared blood flow patterns in a radial artery and a vertebral artery, as representatives of a peripheral artery and an artery supplying the brain. Due to reasons explained below, publication of this pilot experiment was postponed until now.

\section{Case Presentation}

When we carried out the heart catheterization study and the present pilot experiment in 1980, research ethical committees did not exist in our country and ethical approval was not required. ${ }^{6,7}$ A 39 years old male with good knowledge of the study protocol, consented to act as test person. We used the same ergometer bicycle set-up as employed in the heart catheterization study. A pulsed Doppler ultrasound velocity meter (UNIDOP, University of Oslo, Oslo Norway), was used to determine average blood velocity $(\mathrm{ABV})$ in meter per second $(\mathrm{m} / \mathrm{s})$ in a radial artery and a vertebral artery, respectively, as described previously. ${ }^{8}$ When the test person cycled at the same constant workload (163 Watt) as in our previous study, we determined $\mathrm{ABV}$ in the right radial artery by means of a $6 \mathrm{MHz}$ hand-held pulsed Doppler ultrasound transducer, and correspondingly, with a $1.5 \mathrm{MHz}$ transducer in the vertebral artery. ${ }^{6,8}, 9$ The transducers were positioned in right angle towards the respective arteries; first while the test subject breathed freely and subsequently while he held his breath and immersed his face into a basin filled with ice cubes in water (AFI). We registered HR from the electrocardiogram (ECG) signals with electrodes placed on the shoulders and the left iliac crest. The test person rested his right forearm on an arm board while one of the investigators searched up the optimal Doppler-ultrasound signals from the radial artery. Without moving his forearm, the test person carefully bent his upper body and started AFI, which lasted as long as he could endure (30-45 seconds), until he carefully withdrew his face and resumed breathing. The ABV measurements were repeated while the test person cycled with the same workload and held his breath without immersing his face, and finally, while breathing via a snorkel during the face immersion.

When assessing the right vertebral artery $\mathrm{ABV}$, the examiner positioned the Doppler ultrasound transducer as described previously. ${ }^{9}$ While the test person cycled at the same constant workload as in the afore mentioned study, velocity meter signals were obtained both before he carefully bent his upper body forward for AFI, during AFI, and shortly after he cautiously withdrew his face from water. $^{6}$

During ergometer cycling with constant workload, the radial artery $\mathrm{ABV}$ presented with respiration-synchronous changes, as shown during the first half a minute in Figure 1A. When steady state was obtained, as judged by HR of 150-160 beats/min, the test person began AFI (arrows indicate start and end), whereupon radial artery ABV signaling fell by $85 \%$, in parallel with a $58 \%$ decline in HR (Figure 1B), as compared with the preceding period when he breathed freely. Subsequently, the test person held his breath without face immersion while cycling at the same workload. This resulted in similar patterns of decreased ABV (Figure 2A) and HR (Figure 2B), although developing at a slower pace. Of note, we observed sudden bursts in HR during the bradycardia periods both in Figures 1B and 2B. We also wondered if exposure to icecold water alone would trigger the decreases in radial artery ABV and HR. During exposure to the same workload, the test person underwent a new period of cold water face immersion while breathing through a snorkel. However, this time radial artery ABV fell only transiently and returned to the preceding level before he had withdrawn his face from water (Figure 3A). We observed no concomitant drop in HR (Figure 3B). Finally, we investigated right vertebral artery $\mathrm{ABV}$ at the same workload as used above (Figure 4). During the ergometer cycling, ABV increased from $0.24 \mathrm{~m} / \mathrm{s}$ to $0.40 \mathrm{~m} / \mathrm{s}(67 \%)$ during AFI, and subsequently returned to $0.20 \mathrm{~m} / \mathrm{s}$ after AFI. Thus, even during strenuous muscular exercise, AFI appears to redistribute blood flow from the periphery to the brain circulation.

\section{Discussion}

When the test person cycled at constant workload, radial artery $\mathrm{ABV}$ dropped by $85 \%$ and vertebral artery $\mathrm{ABV}$ increased by $67 \%$ during $A F I$ in ice-cold water. 

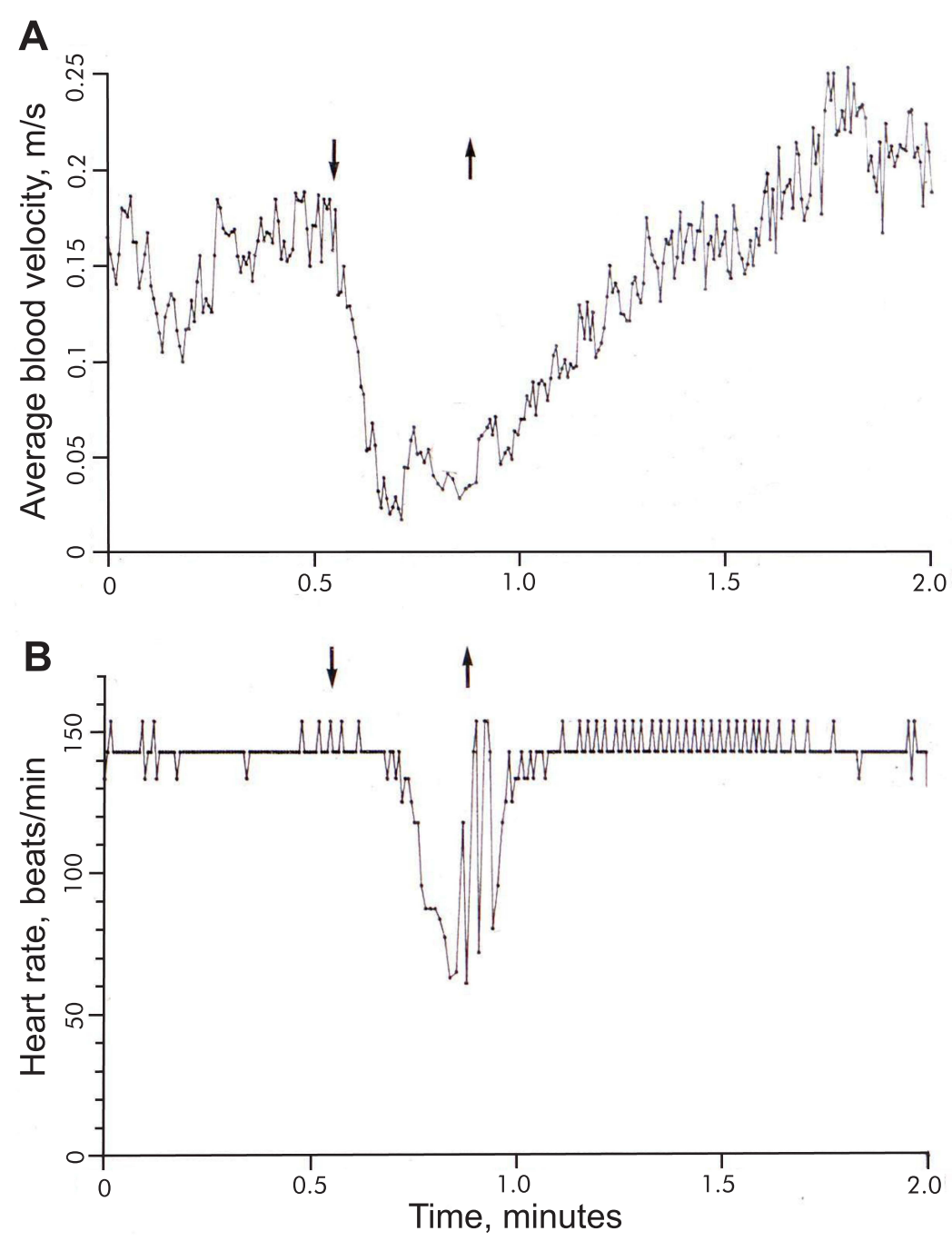

Figure I Average blood velocity in right radial artery (A) and heart rate (B) vs time of a test person during steady state ergometer cycling. Arrows indicate start and stop of a period of apnea and facial immersion (AFI) in ice-cold water.

Concomitantly, HR fell by almost $60 \%$. The fall in radial artery $\mathrm{ABV}$ is consistent with reduced forearm blood flow, as determined by venous occlusion plethysmography during breath hold and whole body immersion at rest. ${ }^{5}$ When the test person performed breath holding without immersing face in cold water, both radial artery $\mathrm{ABV}$ and $\mathrm{HR}$ fell more slowly. Moreover, when he breathed through a snorkel during face immersion in cold water while maintaining unaltered workload, his radial artery ABV dropped only transiently with no concomitant fall in HR. This indicates that despite cycling at a relatively high muscular workload, the "cold shock response" potentiates redistribution of arterial blood from the periphery to the brain, as recently reviewed by Shattock and Tipton. ${ }^{10}$

We cannot argue that the AFI-associated redistribution of blood flow was entirely due to the so-called diving response. In the heart catheterization study, we measured end-expiratory (Alveolar, A) partial pressures of carbon dioxide $\left(\mathrm{P}_{\mathrm{A}} \mathrm{CO}_{2}\right)$ and oxygen $\left(\mathrm{P}_{\mathrm{A}} \mathrm{O}_{2}\right)$, by mass spectrometry following AFI (as median and range) to $10.0(9.1-10.2) \mathrm{kPa}$ and $5.6(5.4-5.7) \mathrm{kPa}$, respectively, when exercising at the same workload. ${ }^{6}$ Since $\mathrm{P}_{\mathrm{A}} \mathrm{CO}_{2}$ increased by nearly $100 \%$ at the end of AFI, the increase in vertebral artery $\mathrm{ABV}$ might have resulted, from increased $\mathrm{P}_{\mathrm{A}} \mathrm{CO}_{2}$, as reported by Hauge et al some years ago. ${ }^{9}$ In addition to the effect on vertebral artery $\mathrm{ABV}$ of almost a doubling of $\mathrm{P}_{\mathrm{A}} \mathrm{CO}_{2}$ as compared to normal, the concomitant effect of a more than $50 \%$ decrease in $\mathrm{P}_{\mathrm{A}} \mathrm{O}_{2}$ also might have contributed to the increase in vertebral artery $\mathrm{ABV}$, since a decrease in $\mathrm{P}_{\mathrm{A}} \mathrm{O}_{2}$ below $6.7 \mathrm{kPa}$ has been suggested to cause cerebral vasodilation. ${ }^{11,12}$ 

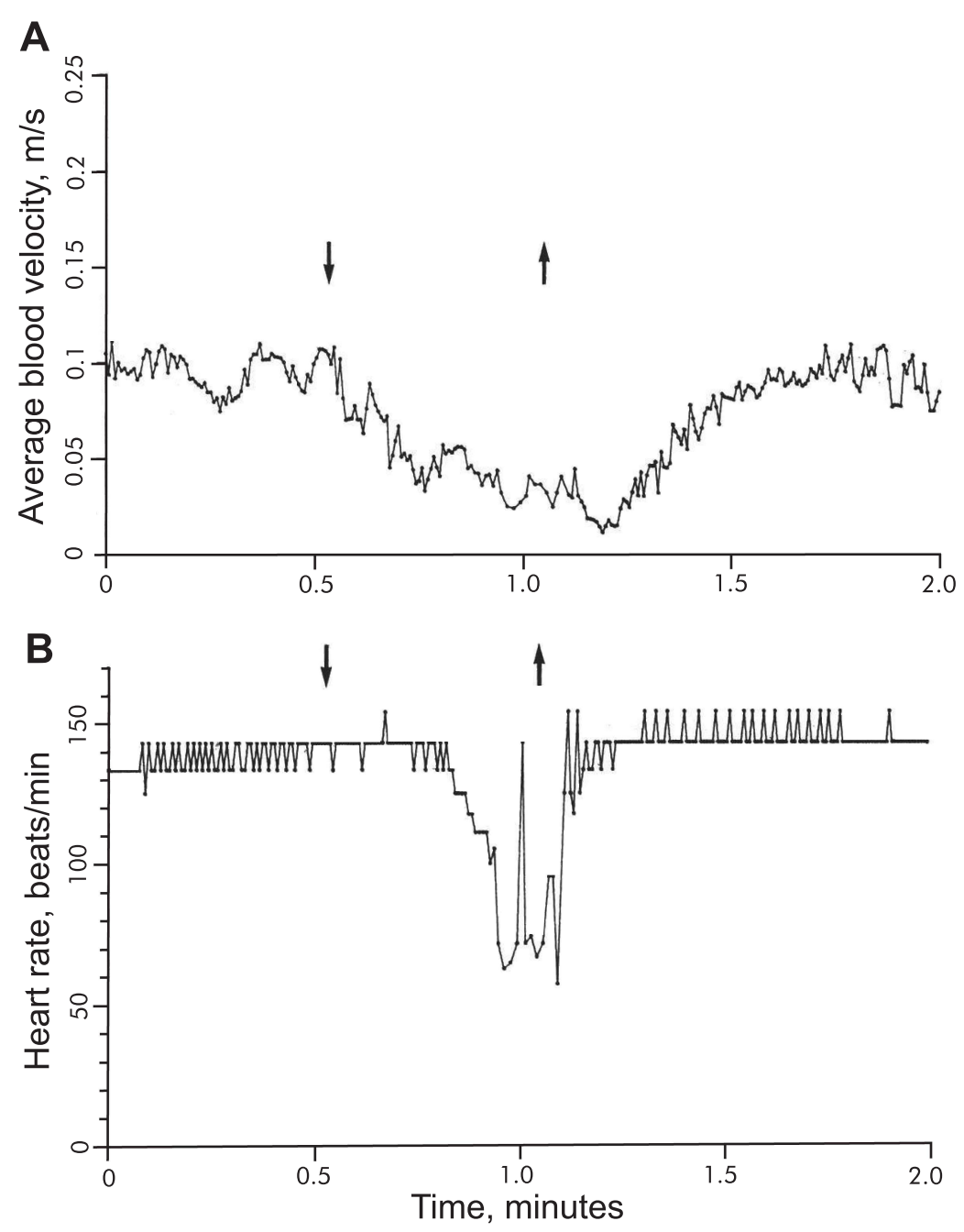

Figure 2 Average blood velocity in right radial artery $(\mathbf{A})$ and heart rate $(\mathbf{B})$ vs time of a test person during steady state ergometer cycling. Arrows indicate start and stop of an apnea period without face immersion in ice-cold water.

We found no study similar to the present one until 2009 when Kjeld et al reported nearly a $180 \%$ increased ABV in the middle cerebral artery (MCA) during periods of apnea at rest. Concomitantly, arterial $\mathrm{PCO}_{2}\left(\mathrm{PaCO}_{2}\right)$ increased from 4.2 to $6.7 \mathrm{kPa}$. While the investigators cycled at a workload of 100 Watt, ABV in MCA increased by $105 \%$ during breath holding, and by a further $9 \%$ during AFI in water of $10^{\circ} \mathrm{C} .{ }^{13}$ This finding is consistent with the present observation of increased $\mathrm{ABV}$ in the vertebral artery during AFI in ice-cold water while the test person cycled at a slightly higher workload.

It is an interesting thought that the central blood redistribution, as demonstrated here, might lead both to increased cerebral oxygen supply, and possibly also contribute to more rapid cooling of the brain in conditions with impeded respiration. In victims of drowning or burial by avalanche, redirecting oxygenated blood to the brain, which is the most sensitive organ to lack of oxygen, probably increases the chance of surviving. Moreover, a faster brain cooling, as demonstrated during experimental dives in seals, might reduce local metabolism, thereby making the oxygen supplied during the cardiac compression to last longer and protect the brain more efficiently against anoxic injury. ${ }^{14}$ Although we consider the duration of AFI as being too short for a brain cooling effect during this experiment, the mechanism might have played a role for the outcome of a 40 year old man, who fell into a ditch without being able to rescue himself. He suffered cardiac arrest when the paramedics lifted him into the ambulance and was subjected to cardiac compression for $4 \frac{1}{2}$ hrs during transfer (by ambulance helicopter) to a tertiary hospital where he was successfully resuscitated with cardiopulmonary bypass 

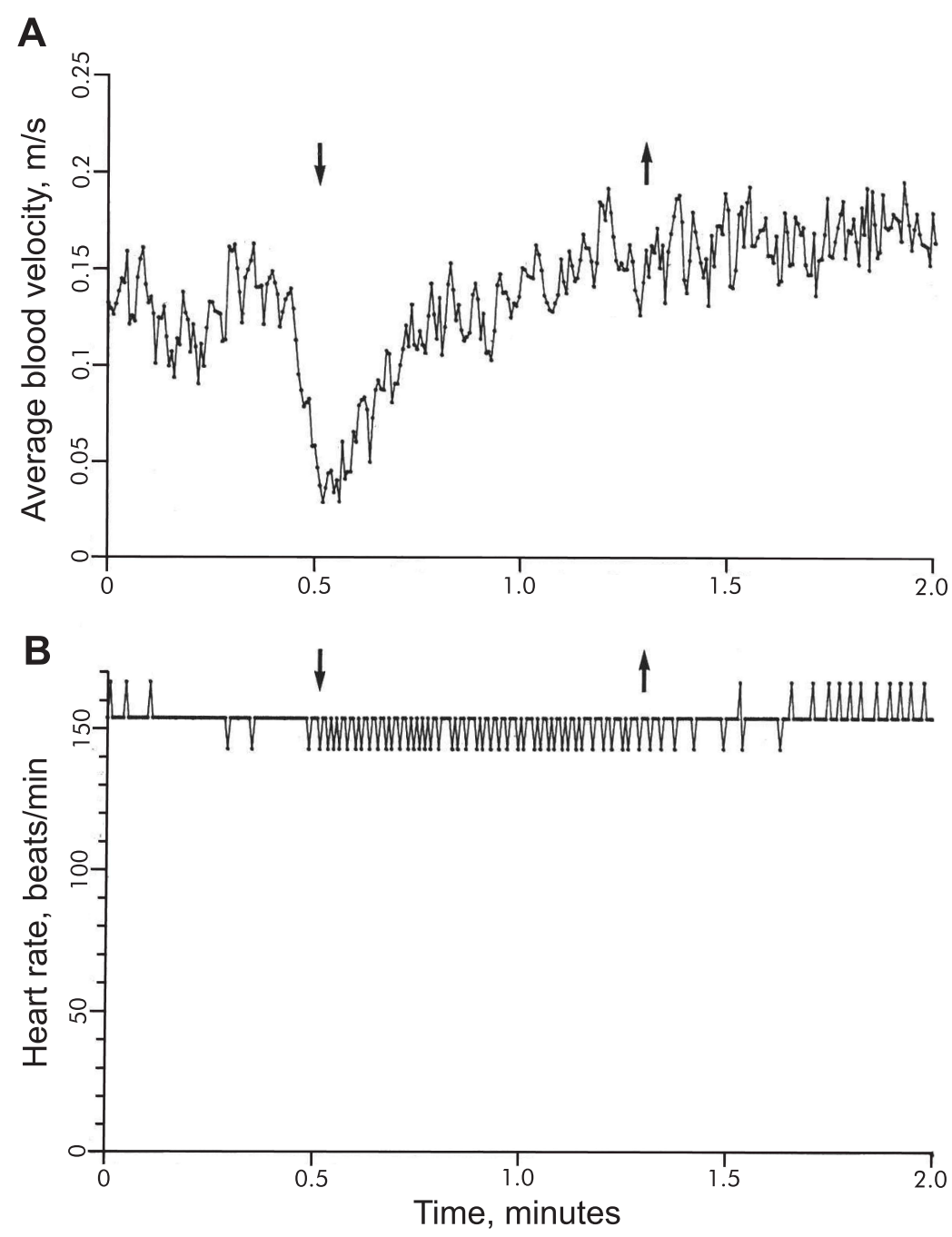

Figure 3 Average blood velocity in the right radial artery (A) and heart rate (B) vs time of a test person during steady state ergometer cycling. Arrows indicate start and stop of a period of facial immersion in ice-cold water during which the test person breathed via a snorkel.

(CPB) from a body core temperature of $25{ }^{\circ} \mathrm{C}$. He was weaned off $\mathrm{CPB}$ nearly seven hrs after $\mathrm{CA}$ and recovered without sequela. $^{15}$

Our findings have limitations aside from the fact that we performed the experiments only on one person more than 40 years ago. Initially, we interpreted the outbreaks of increased HR during AFI (Figures 1B and 2B) as technical errors. However, when the test person was examined for paroxystic atrial fibrillation (year 2002), he presented with episodes of ectopic ventricular beats on the electrocardiogram (ECG) during ergometer bicycling. In 2012, Shattock and Tipton introduced the concept "autonomic conflict", which is a potential mechanism of arrhythmia and a frequent cause of unexpected deaths in cold water. A variety of arrhythmias have been reported, such as atrioventricular blocks, atrial fibrillation, ectopic ventricular beats and prolonged QT times. Therefore, in retrospect, we have reason to believe that ectopic ventricular beats due to "autonomic conflict", rather than technical errors, might have contributed to the outbreaks of tachycardia. $^{10}$

Limitations associated with conversion from $\mathrm{ABV}$ to blood flow were thoroughly studied by previous investigator. ${ }^{8,9,11,16}$ However, we cannot exclude the possibility that a Valsalva maneuver might have contributed to reduce venous return, and consequently, also to decrease $\mathrm{CO}$ and lower radial artery $\mathrm{ABV}$ during breath-holding since the test person changed position from sitting half upright to bending horizontally during AFI. Moreover, a change in arterial transvascular pressure cannot be excluded. However, since central venous pressure also might have increased correspondingly, the driving pressure gradient should remain (grossly) unchanged. This applies particularly to 


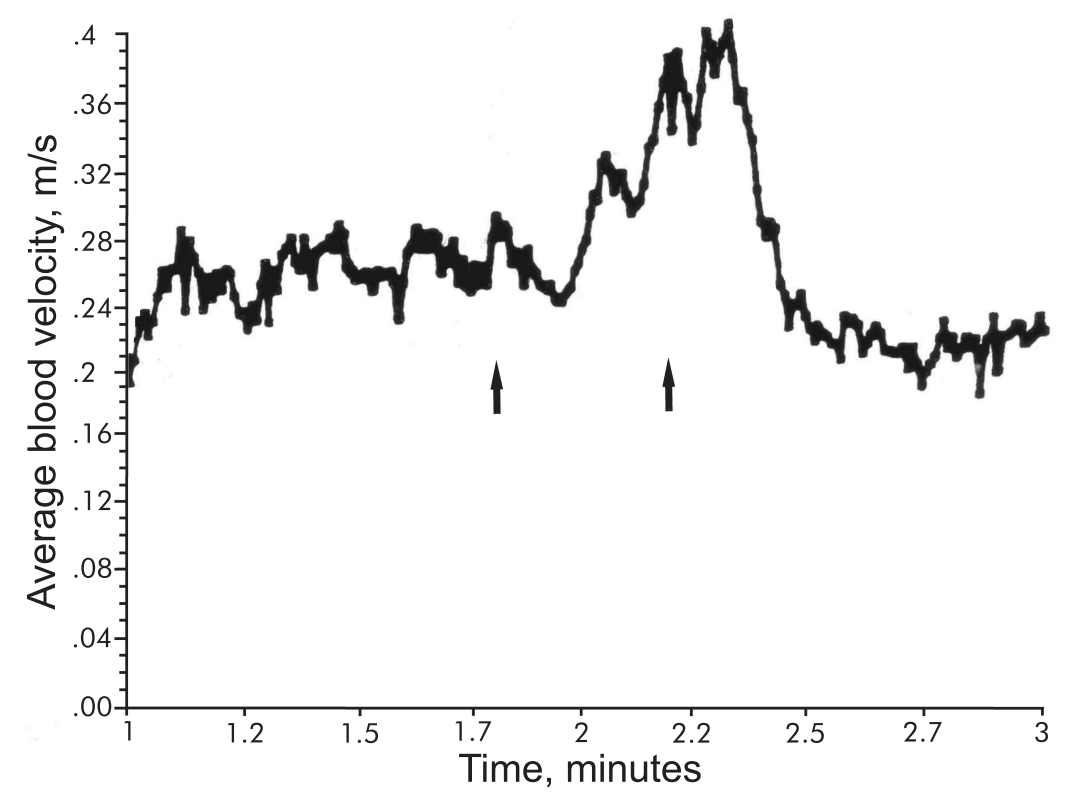

Figure 4 Average blood velocity in the right vertebral artery vs time of a test person during steady state ergometer cycling. Arrows indicate start and stop of a period of apnea and face immersion (AFI) in ice-cold water.

the vertebral artery since hydrostatic pressure changes secondary to positioning changes expectedly were more pronounced in that vessel as compared with that of the radial artery. Since mean cerebral perfusion pressure, determined as the difference between mean systemic arterial pressure and right atrial pressure varied between $93 \mathrm{~mm} \mathrm{Hg}$ and $128 \mathrm{~mm} \mathrm{Hg}$ (during ergometer cycling and AFI) for the same experimental person in the heart catheterization study, we assume that cerebral perfusion pressure remained within the limits of cerebral blood flow autoregulation also in the present study. ${ }^{6,17} \mathrm{We}$ also admit as limitations that we neither determined vertebral artery ABV during AFI in thermo-neutral water, nor during breath hold without face immersion, nor during face immersion while breathing via a snorkel. Previous investigators have shown that cerebral perfusion is maintained and even enhanced during cold stimulation of the face. ${ }^{18}$

Remaining in a position enabling free breathing while the body gradually cools until the heart stops, would seem advantageous for successful reanimation after drowning or avalanche. However, when breathing is inhibited, our findings suggest that apnea combined with exposure of the face to cold water will contribute both to decreased HR and redistribution of blood from the periphery to the central circulation, as shown in diving mammals. Our findings are supported by a report of young victims of drowning who were successfully resuscitated despite the fact that they were found floating with their faces submersed and time to first contact with emergency service was median 121 (108-169) minutes. ${ }^{19}$

\section{Conclusion}

We suggest that the "diving response" combined with cold water stimulation of facial cold thermo-receptors and emerging hypercapnia and hypoxia increases cerebral blood flow during AFI. These mechanisms may well contribute to maintain cerebral oxygenation, and possibly, also induce faster brain cooling before cardiac arrest occurs in conditions of sudden airway blockade, thus, increasing the chances of surviving without neurological sequelae.

\section{Ethics and Consent}

When we carried out the heart catheterization study ${ }^{6}$ and the present pilot experiment in 1980, medical ethics committees were not established in our country and ethical approval was not required. ${ }^{7}$

\section{Consent for Publication}

The person who volunteered to serve as test person consented in publishing case details and the accompanying images.

\section{Acknowledgments}

The authors thank the Publication fund of UiT, The Arctic University of Norway, N-9037 Tromsø, Norway, for covering the publication charges. 


\section{Disclosure}

The authors declare that the research was conducted in the absence of any commercial or financial relationship that could be construed as a potential conflict of interest.

\section{References}

1. Siebke H, Rod T, Breivik H, Lind B. Survival after 40 minutes; submersion without cerebral sequeae. Lancet. 1975; (7919):1275-1277. doi:10.1016/S0140-6736(75)92554-4

2. Althaus U, Aeberhard P, Schupbach P, Nachbur BH, Muhlemann W. Management of profound accidental hypothermia with cardiorespiratory arrest. Ann Surg. 1982;195(4):492-495. doi:10.1097/00000658198204000-00018

3. Fodden DI, Crosby AC, Channer KS. Doppler measurement of cardiac output during cardiopulmonary resuscitation. J Accid Emerg Med. 1996;13(6):379-382. doi:10.1136/emj.13.6.379

4. Brick I. Circulatory responses to immersing the face in water. $J$ Appl Physiol. 1966;21(1):33-36. doi:10.1152/jappl.1966.21.1.33

5. Campbell LB, Gooden BA, Horowitz JD. Cardiovascular responses to partial and total immersion in man. J Physiol. 1969;202(1):239-250. doi:10.1113/jphysiol.1969.sp008807

6. Bjertnaes L, Hauge A, Kjekshus J, Soyland E. Cardiovascular responses to face immersion and apnea during steady state muscle exercise. A heart catheterization study on humans. Acta Physiol Scand. 1984;120(4):605-612. doi:10.1111/j.1748-1716.1984.tb07427.x

7. Lahlum E, Ruyter KW. The establishment of ethical committees for medical research. Tidsskr nor Laegeforen. 2012;132(1213):1486-1489. doi:10.4045/tidsskr.12.0311

8. Thoresen M, Walløe L. Skin blood flow in humans as a function of environmental temperature measured by ultrasound. Acta Physiol Scand. 1980;109(3):333-341. doi:10.1111/j.1748-1716.1980.tb06604.x

9. Hauge A, Thoresen M, Walloe L. Changes in cerebral blood flow during hyperventilation and CO2-breathing measured transcutaneously in humans by a bidirectional, pulsed, ultrasound Doppler blood velocitymeter. Acta Physiol Scand. 1980;110(2):167-173. doi:10.1111/j.1748-1716.1980.tb06647.x
10. Shattock MJ, Tipton MJ. 'Autonomic conflict': a different way to die during cold water immersion? J Physiol. 2012;590(14):3219-3230. doi:10.1113/jphysiol.2012.229864

11. Ellingsen I, Hauge A, Nicolaysen G, Thoresen M, Walløe L. Changes in human cerebral blood flow due to step changes in $\mathrm{PAO}_{2}$ and $\mathrm{PACO}_{2}$. Acta Physiol Scand. 1987;129(2):157-163. doi:10.1111/ j.1748-1716.1987.tb08054.x

12. Masamoto K, Tanishita K. Oxygen transport in brain tissue. J Biomech Eng. 2009;131:7. doi:10.1115/1.3184694

13. Kjeld T, Pott FC, Secher NH. Facial immersion in cold water enhances cerebral blood velocity during breath-hold exercise in humans. J Appl Physiol. 2009;106(4):1243-1248. doi:10.1152/ japplphysiol.90370.2008

14. Blix AS, Walløe L, Messelt EB, Folkow LP. Selective brain cooling and its vascular basis in diving seals. J Exp Biol. 2010;213(Pt 15):2610-2616. doi:10.1242/jeb.040345

15. Mark E, Jacobsen O, Kjerstad A, et al. Hypothermic cardiac arrest far away from the center providing rewarming with extracorporeal circulation. Int J Emerg Med. 2012;5(1):7. doi:10.1186/ 1865-1380-5-7

16. Guldvog I, Kjaernes M, Thoresen M, Walloe L. Blood flow in arteries determined transcutaneously by an ultrasonic doppler velocitymeter as compared to electromagnetic measurements on the exposed vesels. Acta Physiol Scand. 1980;109(2):211-216. doi:10.1111/j.1748-1716.1980.tb06588.x

17. Lassen NA. Cerebral blood flow and oxygen consumption in man. Physiol Rev. 1959;39(2):183-238. doi:10.1152/physrev.1959.3 9.2.183

18. Brown CM, Sanya EO, Hilz MJ. Effect of cold face stimulation on cerebral blood flow in humans. Brain Res Bull. 2003;61(1):81-86. doi:10.1016/S0361-9230(03)00065-0

19. Wanscher M, Agersnap L, Ravn J, et al. Outcome of accidental hypothermia with or without circulatory arrest: experience from the Danish Praesto Fjord boating accident. Resuscitation. 2012;83 (9):1078-1084. doi:10.1016/j.resuscitation.2012.05.009

\section{Publish your work in this journal}

The International Medical Case Reports Journal is an international, peer-reviewed open-access journal publishing original case reports from all medical specialties. Previously unpublished medical posters are also accepted relating to any area of clinical or preclinical science. Submissions should not normally exceed 2,000 words or 4 published pages including figures, diagrams and references. The manuscript management system is completely online and includes a very quick and fair peer-review system, which is all easy to use. Visit http://www.dovepress.com/testimonials.php to read real quotes from published authors. 